LIVER FIBROSIS

\title{
Progression of hepatic fibrosis in patients with hepatitis C: a prospective repeat liver biopsy study
}

\section{S D Ryder, on behalf of the Trent Hepatitis C Study Group}

Gut 2004;53:451-455. doi: 10.1136/gut.2003.021691

Background: The natural history of hepatitis $\mathrm{C}$ virus $(\mathrm{HCV})$ infection remains uncertain. Previous data concerning rates of progression are from studies using estimated dates of infection and single liver biopsy scores. We prospectively studied the rate of progression of fibrosis in $\mathrm{HCV}$ infected patients by repeat liver biopsies without intervening treatment.

Patients: We studied $214 \mathrm{HCV}$ infected patients (126 male; median age 36 years (range 5-8)) with predominantly mild liver disease who were prospectively followed without treatment and assessed for risk factors for progression of liver disease. Interbiopsy interval was a median of 2.5 years. Paired biopsies from the same patient were scored by the same pathologist.

Results: Seventy of 219 (33\%) patients showed progression of at least 1 fibrosis point in the Ishak score; 23 progressed at least 2 points. Independent predictors of progression were age at first biopsy and any

Correspondence to: Dr S D Ryder, Queen's Medical Centre, University Hospital, Nottingham NG7 2UH, UK; stephen.ryder@ mail.qmcuh-tr.trent.nhs.uk

Accepted for publication 8 July 2003 fibrosis on first biopsy. Factors not associated with progression were: necroinflammation, duration of infection, alcohol consumption, alanine aminotransferase levels, current or past hepatitis B virus infection, ferritin, HCV genotype, and steatosis or iron deposition in the initial biopsy.

Conclusions: One third of patients with predominantly mild hepatitis C showed significant fibrosis progression over a median period of 30 months. Histologically, mild hepatitis $C$ is a progressive disease. The overall rate of fibrosis progression in patients with hepatitis $C$ was low but increased in patients who were older or had fibrosis on their index biopsy. These data suggest that HCV infection will place an increasing burden on health care services in the next 20 years.
$\mathrm{H}$ epatitis C virus (HCV) was described in $1989^{1}$ and is a significant cause of chronic liver disease. A number of factors have been suggested to influence progression of liver disease towards cirrhosis. Data are from studies which are predominantly retrospective or have uncertain inclusion criteria; many are from tertiary referral centres which may have a bias towards more severe liver disease. The largest study of the natural history of liver fibrosis in HCV infection ${ }^{2}$ relies on a single liver biopsy fibrosis score and an estimated duration of infection, thus making assumptions that the date of infection was reliable and that liver fibrosis progresses at a linear rate.

Treatment for HCV infection remains complex, is costly, has substantial side effects, and even with improved regimens only a $55 \%$ prospect of success. ${ }^{3}$ The majority of patients with HCV have mild liver disease on biopsy. Hence reliable data on the rates and risk factors for progression of liver fibrosis are essential for the development of a rational treatment strategy.

This study aimed to determine the factors leading to histological progression of fibrosis in a cohort of patients with HCV infection with paired liver biopsy samples taken after a time interval, with no intervening therapy.

\section{PATIENTS AND METHODS}

The Trent HCV Study Group is a collaborative multidisciplinary group whose aim is to produce both research into the pathogenesis of disease and clinical guidelines for therapy in HCV infection. Within the Trent Health Care Region, covering a population of 5.1 million, the majority of individuals found to be HCV antibody positive are referred to a clinician within the group for investigation and follow up. The group has an agreed management protocol, with a decision algorithm which states that therapy should be offered to patients with moderate to severe liver disease, originally taken as a total Knodell score of 6 or more ${ }^{4}$ Any patient with a score below 6, or who declines therapy, is followed prospectively and a repeat liver biopsy recommended after a two year interval. Patients with known human immunodeficiency virus infection, coagulation disorders, and those on renal replacement therapy were excluded from the cohort as the natural history may differ in these groups. The Trent HCV study started in 1991.

\section{Patients included in the study cohort}

As of December 2000, 1978 patients had been entered into the Trent HCV Study Group database. ${ }^{4}$ Full ethics approval was obtained for the study. Liver biopsy had been undertaken in 841 HCV RNA positive patients. A total of 261 patients had received treatment with interferon after their initial liver biopsy. Of the 580 untreated patients, 216 have presently less than two years of follow up and have not yet received a second liver biopsy, 40 (7\%) have declined further biopsy, and 73 (13\%) have been lost to follow up. In 32 patients, one or other biopsy was either unobtainable or deemed too small to score. Five patients with paired samples had cirrhosis on the first biopsy and therefore could not progress. Biopsy scores and data collection are complete in 214 patients who form the group reported herein.

\section{Variables measured}

The genotype of the infecting HCV was assessed by restriction fragment length polymorphism assay ${ }^{5}$ or, more recently, by a commercially available line probe assay (Inno-LiPa).

Abbreviations: $\mathrm{HCV}$, hepatitis $C$ virus; $H B V$, hepatitis $B$ virus; $H B s A g$, hepatitis B surface antigen; anti-HBc, anticore hepatitis B antibody; ALT, aminotransferase; IVDU, intravenous drug use 
Hepatitis B virus (HBV) infection was sought by hepatitis B surface antigen ( $\mathrm{HBsAg}$ ) testing and in those negative for HBsAg, anticore antibody (anti-HBc) as evidence of prior infection.

Serum ferritin, and mean and peak alanine aminotransferase levels (ALT) were recorded, together with immunoglobulins and autoantibodies.

At enrolment into the Trent HCV Cohort Study, all patients are asked to complete a detailed risk factor questionnaire which provides information on factors associated with spread of blood borne viruses (intravenous drug use (IVDU), transfusion, sexual history, tattooing). Patient demographic details (age at biopsy, date of first exposure to a known risk factor, sex) were also recorded. At each clinic visit, all patients complete a questionnaire detailing their alcohol consumption over the week prior to clinic attendance.

\section{Histological assessment of liver biopsy samples}

Liver biopsies were all standard percutaneous samples, and the threshold of adequacy for histological assessment was the presence of more than five portal tracts. All biopsy samples were assessed blindly for the study, separate from the clinical assessment made by local histopathologists, by one of three histopathologists (JCEU, AZ, and JW), and scored for fibrosis and necroinflammatory changes (Knodell, Ishak). ${ }^{6}$ Iron stores were scored on Perl's stained sections, with grading from 0 to 4 (4, maximum). Steatosis was scored from 0 (none) to 4 (extensive). The pathologist had no knowledge of data other than the patient identifier and laboratory accession number. Each pair of biopsies was scored by the same pathologist.

\section{Statistical analysis}

SPSS for Windows vl0 was used to produce logistic regression models, with progression of fibrosis by a score of more than 1 stage point as the dependent variable. Terms were kept in the model at $\mathrm{p}<0.1$. For statistics relating to Ishak necroinflammatory score, initial scores were also categorised in groups (scores 0 and 1, scores 2-4, scores 56 , and score 7 or more).

\section{RESULTS}

\section{Patient details}

Of the 214 patients studied, 126 were male (59\%). Median age at the time of initial biopsy was 36 years (range 5-78). A total of $114(52 \%)$ patients admitted to having used injectable drugs and a further 49 (23\%) had received blood components prior to 1991 (when blood donor screening for HCV was introduced in the UK). Where known, viral genotypes were 1 in 73 patients, 2 in 21, 3 in 60, and one each of types 4 and 5. Three patients were HBsAg positive and a further 53 had evidence of previous exposure to HBV (anti-HBc positive). Median duration of infection among patients with a probable date of infection (using the first date of exposure to risk) was 18.9 years (range 4-41). Median interbiopsy interval was 2.5 years (range 1.9-9.4). Seven per cent of all biopsies in this study were scored twice, producing an intraobserver variability of 0.07 for fibrosis and 0.13 for necroinflammation, and an interobserver variability of 0.1 for fibrosis stage and 0.2 for necroinflammatory grade.

\section{Assessment of initial liver biopsy}

The median total Ishak score on the initial biopsy was 3 (range 0-19) (fig 1). The majority of patients (188) had total scores of 6 or less, with fibrosis stage scores of 0 or 1 ( 183 patients; fig 1).
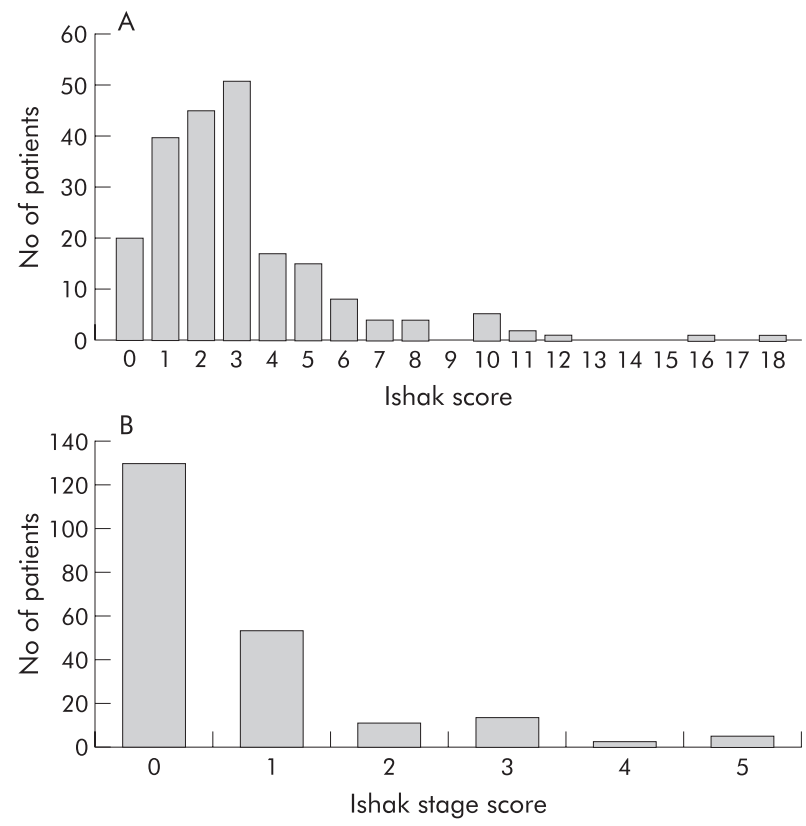

Figure 1 (A) Total (necroinflammatory plus fibrosis) and fibrosis stage (B) Ishak scores on the index liver biopsy.

Progression of disease evident on second liver biopsy Median necroinflammatory grade and fibrosis stage scores progressed between biopsies from 2 to 3 and from 0 to 1 , respectively $(p=0.025$ and $p<0.001)$. Changes in these scores are shown in table 1. Seventy of 214 (33\%) patients had an increase in fibrosis stage score of 1 or more point in Ishak stage (table 1). Twenty three of 210 patients ( $11 \%$ ) (excluding five patients with fibrosis stage 5 or more on the index biopsy who therefore could not progress by more than a single point) showed progression of 2 points or more. Twenty two patients had a reduction in fibrosis score on the second biopsy ( $10 \%), 20$ by 1 point, one by 2 points, and one by 3 points.

\section{Factors related to fibrosis progression between biopsies 1 and 2}

For the purposes of analysis of fibrosis progression, patients were considered to have any progression if the score increased by 1 point or more and serious progression if the score increased by 2 points. The comparator groups for the analysis were a combination of patients with any fibrosis regression and those with no change in fibrosis for analysis of 1 point progression. For the severe ( 2 point) progressors, the comparator group was patients with fibrosis regression, stable fibrosis, and 1 point progression.

The results of univariate analysis of factors influencing severe fibrosis progression (2 points in Ishak stage) are shown in table 2. Age at biopsy (45.6 $v 36.2$ years; $\mathrm{p}<0.001)$, estimated age at infection (27.4 $v 18.7$ years; $\mathrm{p}=0.04)$, necroinflammatory score on biopsy 1 (median $4 \quad v \quad 2$; $\mathrm{p}=.007)$, and fibrosis score on biopsy $\mathrm{l}$ (16/23 progressors $v 64 / 187$ non-progressors; $\mathrm{p}=0.001$ ) all predicted progression while sex, alcohol consumption, steatosis and siderosis on biopsy 1, ALT, and HCV genotype did not. Markers of HBV infection were not statistically significant as a risk factor for progression although there was an excess of anticore antibody positive patients in the progressor group. Interval between biopsy (taken as $0-2$ years, $2-4$ years and $>4$ years) was also not a significant factor predicting fibrosis, probably because $88 \%$ of all second biopsies occurred between 2.5 and 3.5 years after the index biopsy. 
Table 1 Change in Ishak scores for fibrosis stage and necroinflammatory change

\begin{tabular}{|c|c|c|c|c|c|c|c|c|c|c|c|}
\hline & \multicolumn{11}{|c|}{ Change in score } \\
\hline & $\geqslant 4$ & -3 & -2 & -1 & 0 & 1 & 2 & 3 & 4 & 5 & $>5$ \\
\hline Grade & 5 & 6 & 13 & 46 & 54 & 40 & 26 & 11 & 7 & 4 & 2 \\
\hline Stage & 0 & 0 & 2 & 20 & 122 & 47 & 12 & 5 & 3 & 3 & N/A \\
\hline
\end{tabular}

Number of patients with change in fibrosis stage and necroinflammatory grade scores between paired liver biopsies in 214 untreated patients with hepatitis C.

The results of multivariate analysis are shown in table 3. The most significant independent risk factors for severe progression of fibrosis by 2 points or more were age at biopsy and the presence of any fibrosis on the index biopsy. No other variable reached independent statistical significance. Identical results were obtained if significant progression in fibrosis was defined as 1 point in the Ishak score.

Immunoglobulin $\mathrm{G}$ levels were elevated in 16 patients but unrelated to fibrosis progression $(\mathrm{p}=0.81)$. Five patients had low titre $(<\mathrm{l} / 50)$ positive antinuclear antibody levels and three had low titre $(<\mathrm{l} / 100)$ antismooth muscle antibodies; again, neither was associated with fibrosis progression. Mode of transmission (IVDU $v$ other) had no association with progression.

No significant differences were obtained relating to risk of progression of fibrosis if Knodell scores for fibrosis were used rather than the Ishak fibrosis score. A very strong correlation existed between the two scoring systems $(p=0.0001$, data not shown).

Forty six patients had persistently normal ALT values. Overall, fibrosis progression was seen in $10(22 \%)$ of these patients (five by 1 Ishak stage, five ( $12 \%$ ) by more than 1 ). While indicating a trend for disease progression to be less in this subgroup, these values were not significantly different from those for patients with abnormal ALT values where 59/ $162(36 \%)$ patients progressed (41 by l stage, 18 (12\%) by more than 1).

The two independent risk factors for progression of fibrosis (table 3) both conferred a significant risk of progression. Table 4 indicates the numbers of patients whose fibrosis did or did not progress, according to whether they possessed none or both of the two risk factors (age over 35 years and fibrosis on the index biopsy). None of 59 patients in the former group had fibrosis progression by 2 Ishak stages compared with $14 / 65$ (22\%) patients in the latter group.

\section{DISCUSSION}

This large prospective liver biopsy based study of fibrosis progression in chronic HCV infection demonstrates that even in patients with apparently mild liver disease at presentation, progression of fibrosis does occur in a significant proportion $(70 / 214,33 \%)$ in less than three years.

Most previous studies of the natural history of hepatitis $\mathrm{C}$ infection have been retrospective. ${ }^{289}$ The largest study ${ }^{2}$ used a single liver biopsy with an estimated duration of infection to calculate a fibrosis progression rate. This approach has inherent error, infection duration relying predominantly on date of first use of injectable drugs. In addition, $17 \%$ of patients in that study had cirrhosis on initial biopsy and fibrosis cannot be graded further than cirrhosis. It is well known that asymptomatic cirrhosis can be present for many years, thus inclusion of such patients will underestimate fibrosis progression. Use of a single biopsy and calculation of progression rates also assumes linear progression through all stages of infection, an assumption which has no clear evidence base.

There are other prospective series of patients in the literature. ${ }^{10-12}$ The largest of these studied 123 patients ${ }^{12}$ with a mean interval between biopsies of 44 months. None of these studies has details of the total patient population and how they were selected, which limits the significance of their conclusions. The major factor identified as increasing fibrosis risk in these studies was necroinflammation, a factor not significant in studies of single biopsies with an estimated date of infection..$^{13}$ In univariate analysis, our data showed that necroinflammatory grade score in the index biopsy predicted fibrosis progression but this was not an independent factor when entered into the multivariate analysis. The necroinflammatory grade in this cohort of patients was modest ( $>5$ in only 26 patients) on the index biopsy but showed a significant rise in the second biopsy. This is the first study to show that necroinflammation increases over time.

Table 2 Univariate analysis of factors influencing severe (2 points or more) fibrosis progression

\begin{tabular}{|c|c|c|c|}
\hline Demographic factor & Progressed $(n=23)$ & No progression $(n=187)$ & ${ }^{*} \mathrm{p}$ Value \\
\hline \multicolumn{4}{|l|}{ Sex } \\
\hline Male & 13 & 109 & \\
\hline Female & 10 & 78 & NS \\
\hline Alcohol (units/week)† & 4.5 & 5.0 & NS \\
\hline Age at index biopsy (y) $\dagger$ & 45.6 & 36.2 & 0.001 \\
\hline Age at infection $(y) \dagger$ & 27.4 & 18.7 & 0.05 \\
\hline Duration of infection $(y) \dagger$ & 24.7 & 18.7 & 0.04 \\
\hline \multicolumn{4}{|l|}{ Route of transmission } \\
\hline Injecting drug use & 13 & 100 & \\
\hline Other & 10 & 77 & NS \\
\hline
\end{tabular}

Risk factors for fibrosis progression in patients with hepatitis $\mathrm{C}$ with severe progressive (2 points or more) or nonprogressive liver fibrosis (regression of fibrosis, no progression of fibrosis, and 1 point progression of fibrosis), between biopsies 1 and 2 .

No data available on risk factors for 10 patients, hepatitis B virus markers for 11 patients, aminotransferase levels for one patient, and hepatitis $C$ virus genotypes for 58 patients.

* $\mathrm{p}$ values for univariate analysis.

†Median values.

${ }^{* *} \chi^{2}$ for linear trend. 
Table 3 Multivariate analysis of factors influencing fibrosis progression: independent risk factors for progression of fibrosis

\begin{tabular}{llll}
\hline Factor & Adjusted RR & $\mathbf{9 5 \%} \mathbf{C l}$ & $\mathbf{p ~ V a l u e ~}$ \\
\hline Fibrosis & 1.93 & $3.38(1.3-9.0)$ & 0.014 \\
Age at biopsy & 1.08 & $1.03-1.11$ & 0.001 \\
\hline
\end{tabular}

$\mathrm{RR}$, relative risk; $95 \% \mathrm{Cl}, 95 \%$ confidence interval.

There was no suggestion in our study that higher ALT values carry an increased risk of fibrosis progression, a finding contrary to another twin biopsy study. ${ }^{12}$ In this cohort, ALT values varied over time in most individuals, and a single, even marked, elevation in ALT value carries little value in that individual as a predictor of fibrosis.

Our study avoided the pitfall of estimated duration of infection by prospectively following a relatively large group of patients selected to have mild or moderate liver disease. In addition, serial data were collected on factors such as alcohol intake and transaminase values, which have not been available previously. The Trent Hepatitis C cohort is likely to be representative of the UK population, as there is no tertiary referral bias in this group and all patients included and excluded from the study were known.

The rate of progression of hepatic fibrosis in this cohort was variable, the most rapid progression being from a fibrosis score of 0 to cirrhosis in less than two years, but the majority of patients showed no progression. If a linear rate of progression is assumed from the time of infection to the development of cirrhosis, the rate of progression overall was 0.17 Ishak fibrosis points per year. This is similar to previous data $^{21213}$ which suggest progression rates from 0.12 to 0.19 units per year.

This study however provides strong evidence that progression of hepatic fibrosis in hepatitis $\mathrm{C}$ is non-linear. The most significant risk factors were age at biopsy, rather than duration of infection, and the presence of fibrosis on the index biopsy. This suggests that hepatitis $\mathrm{C}$ infection may somehow become more fibrogenic with advancing host age. This may be one explanation for the apparent lack of fibrotic liver disease progression in young women infected with hepatitis C via immunoglobulin anti-D. ${ }^{14}$ There are emerging data from the post-transplant setting which suggest that this may be a general effect of the aging liver. Increasing donor age is a significant risk factor for severe post-transplant recurrence of hepatitis C. ${ }^{15}{ }^{16}$ The potential mechanisms for this accelerated organ damage are not known.

All paired liver biopsies in this study were assessed by a single histopathologist, and three experienced liver histopathologists were involved in this study. Intraobserver variation between assessments of the same sample is less for fibrosis stage than for necroinflammatory grade, ${ }^{17}$ and in this study was low. Regression of fibrosis was seen in $10 \%$ of patients in this study, presumably due to a combination of observer error in interpretation and sampling variation. Fibrosis progressed in 33\%.

Previous studies have emphasised the role of male sex and high alcohol consumption, a combination of these factors doubling the rate of fibrosis. ${ }^{2}$ This was not seen here. Overall alcohol consumption in the study group was however very low, as most patients willing to attend regularly and to have repeat liver biopsies take notice of medical advice and consume little alcohol. It is of note that in patients who present with significant liver disease in our cohort, alcohol consumption is much higher that that seen in this follow up study of patients presenting with mild disease. ${ }^{4}$ This suggests that alcohol is an important cofactor at presentation, but if alcohol consumption is reduced, prior fibrosis and age become the most important predictors of severe disease.

Several recent studies have suggested that the presence of steatosis in liver biopsies in hepatitis C may predict progression. ${ }^{18}$ Fibrosis has been correlated with steatosis and body mass index, ${ }^{19}$ and steatosis has been linked with increased hepatitis $\mathrm{C}$ core protein expression. ${ }^{20} \mathrm{~A}$ recent small study in patients with HCV infection showed weight loss reduced both steatosis and fibrosis. ${ }^{21}$ Our data showed that steatosis did not increase the risk of fibrosis progression.

There is evidence of viral interference in livers containing replicating $\mathrm{HBV}$ and HCV. ${ }^{22}{ }^{23}$ There is evidence that HBsAg carriage alone may enhance fibrosis in HCV positive patients. ${ }^{24}{ }^{25}$ The presence of occult HBV infection in patients with HCV infection has been correlated with increased histological severity scores. ${ }^{26-28}$ This study showed no significant association of anti-HBc with increased fibrosis progression although the increased proportion of anti-HBc positive patients in the progressor group is worthy of further study.

Genotypes 1 and 3 predominated in our cohort, a distribution very similar to those previously reported in the UK. ${ }^{29}$ There was no significant relationship between genotype and risk of development of fibrosis. There are conflicting reports in the literature with regard to this issue..$^{30-33}$ Our data do not suggest that genotype 1 has a significant role in progression of fibrosis although it clearly is an important factor in the prediction of response to therapy. ${ }^{34}$

Iron loading has been the subject of intense study in hepatic fibrosis. Hepatic iron concentrations are elevated in hepatitis $\mathrm{C}^{35}$ and correlate with necroinflammatory change and increased stellate cell numbers. ${ }^{36}$ Iron loading has been suggested to be a factor in fibrosis progression in hepatitis C. ${ }^{37}$ Our data showed a non-significant increase in serum ferritin $(77 v 105)$ in patients with progression of hepatic fibrosis but histological iron stores assessed on a Perl's stained section did not correlate with increased fibrosis.

We conclude that histologically mild hepatitis $\mathrm{C}$ is a progressive disease. The overall rate of fibrosis progression is low but increased in patients who are older or have fibrosis on their index biopsy. These data suggest that HCV infection will place an increasing burden on health care services in the next 20 years as the population infected with HCV ages.

Table 4 Risk of progression in two groups with and without significant risk factors for progression

\begin{tabular}{lll}
\hline & "Good risk group" & "Poor risk group" \\
& Age $\leqslant \mathbf{3 5}$ y, no fibrosis & Age $>\mathbf{3 5}$, any fibrosis \\
\hline No of patients & 59 & 65 \\
No progression & $48(81 \%)$ & $39(60 \%)$ \\
1 point progression & $11(19 \%)$ & $12(18 \%)$ \\
2 points progression & $0(0 \%)$ & $14(22 \%)$ \\
\hline
\end{tabular}

"Poor risk", age $>35$ years and fibrosis present on the index biopsy.

"Good risk", age $\leqslant 35$ and no fibrosis. 


\section{ACKNOWLEDGMENTS}

This work was supported by a grant from the Department of Health to the Trent HCV Study Group and a grant from Roche Pharmaceuticals.

\section{APPENDIX}

The paper was written by the writing committee, and reviewed and agreed by all members of the Trent HCV Study group

\section{Writing Committee (in alphabetical order)}

WL Irving, DA Jones, KR Neal, SD Ryder, JC Underwood.

\section{Trent HCV Study Group (number of patients from each centre)}

Derby City Hospital (18), Dr JG Freeman, Consultant Gastroenterologist; Grimsby General Hospital, Dr A Naqvi, Consultant Gastroenterologist; Leicester Royal Infirmary (16), Professor KR Nicholson, Professor of Infectious Diseases, Dr M Wiselka, Consultant in Infectious Diseases; Lincoln County Hospital (18), Dr BB Scott, Consultant Gastroenterologist; Nottingham City Hospital (39), Professor RG Finch, Professor of Infectious Diseases, Dr BJ Thomson, Senior Lecturer in Infectious Diseases; Queen's Medical Centre, Nottingham (45), Professor WL Irving, Professor in Virology (Chair), Dr KR Neal, Senior Lecturer in P H Medicine and Epidemiology, Dr SD Ryder, Consultant Hepatologist, Dr A Zaitoun, Consultant Pathologist; National Blood Authority, Trent Centre, Dr V James, Consultant Haematologist, Dr DA Jones, Consultant Haematologist (Secretary); Royal Hallamshire Hospital, Sheffield (51), Dr J Bremner, Consultant Virologist, Sheffield Virology Consortium, Dr D Gleeson, Consultant Hepatologist, Dr S Green, Consultant in Infectious Diseases, Dr G Kudesia, Consultant Virologist, Sheffield Virology Consortium, Dr MW McKendrick, Consultant in Infectious Diseases, Dr R Read, Senior Lecturer in Infectious Diseases, Professor JCE Underwood, Professor of Pathology; St James's University Hospital, Leeds (32), Dr M Davies, Director, Liver Transplant Unit, Dr C Milson, Consultant Hepatologist, Dr J Wyatt, Consultant Pathologist; Seacroft Hospital, Leeds, Dr EJ Minton, Consultant in Infectious Diseases; Scunthorpe General Hospital, Dr J Penstone, Consultant Gastroenterologist; Hepatitis C Specialist Nurses, Mr Ray Poll (Royal Hallamshire Hospital, Sheffield), Mrs Mandy Holliday (City Hospital, Nottingham), Mrs Kate Jack (Leicester Royal Infirmary), Ms Maggie Nicholls (Queen's Medical Centre, Nottingham), Mrs Sue Sheridan (St James University Hospital, Leeds).

\section{REFERENCES}

1 Choo QL, Kuo G, Weiner AJ, et al. Isolation of a cDNA clone derived from a blood-borne non-A, non-B viral hepatitis genome. Science 1989;244:359-62.

2 Poynard T, Bedossa P, Opolon P. Natural history of liver fibrosis progression in patients with chronic hepatitis C. Lancet 1997;349:825-32.

3 Poynard T, Marcellin P, Lee $S$, et al. Randomised trial of interferon alpa $2 \mathrm{~b}$ plus Ribavirin for 48 weeks or for 24 weeks versus interferon alpha $2 b$ plus placebo for 48 weeks for treatment of chronic infection with hepatitis $C$ virus. Lancet 1998;352:1426-32.

4 Mohsen A, The Trent HCV Study Group. The epidemiology of hepatitis C in a UK Health Regional population of 5.12 million. Gut 2001;48:707-13.

5 Harris KA, Gilham C, Mortimer PP, et al. The most prevalent HCVgenotypes in England and Wales are 3a and la. J Med Virol 1999;58:127-31.

6 Knodell RG, Ishak KG, Black WC, et al. Formulation and application of a numerical scoring system for assessing histological activity in asymptomatic chronic active hepatitis. Hepatology 1981;1:431-5.
7 Ishak KG, Baptista A, Bianchi L, et al. Histological grading and staging of chronic hepatitis. J Hepatol 1995;22:696-9.

8 Yano M, Kumada H, Kage M, et al. The long-term pathological evolution of chronic hepatitis C. Hepatology 1996;23:1334-40.

9 Crowe J, Doyle C, Fielding JF, et al. Presentation of hepatitis $C$ in a unique uniform cohort 17 years from inoculation. Gastroenterology 1995; 108:A1054.

10 Fontaine $H$, Nalpas $B$, Poulet $B$, et al. Hepatitis activity index is a key factor in determining the natural history of chronic hepatitis $\mathrm{C}$. Hum Pathol 2001;32:904-9.

11 Lagging LM, Westin J, Svensson E, et al. Progression of fibrosis in untreated patients with hepatitis C virus infection. Liver 2002;22:136-44.

12 Ghany MG, Kleiner DE, Alter $\mathrm{H}$, et al. Progression of fibrosis in chronic hepatitis C. Gastroenterology 2003;124:97-104.

13 Poynard T, Ratziu V, Charlotte F, et al. Rates and risk factors of liver fibrosis progression in patients with chronic hepatitis C. J Hepatol 2001;34:730-9.

14 Kenny-Walsh E. Clinical outcomes after hepatitis $C$ infection from contaminated anti-D immune globulin. Irish Hepatology Research Group. New Engl J Med 1999:340:1228-33.

15 Wali M, Harrison RF, Gow PJ, et al. Advancing donor liver age and rapid fibrosis progression following transplantation for hepatitis C. Gut 2002;51:248-52.

16 Berenguer $M$, Prieto $M$, San Juan $F$, et al. Contribution of donor age to the recent decrease in patient survival among $\mathrm{HCV}$-infected liver transplant recipients. Hepatology 2002;36:202-10.

17 Goldin RD, Goldin JG, Burt AD, et al. Intra-observer and intra-observer variation in the histopathological assessment of chronic viral hepatitis. $J$ Hepatol, 1996:25:649-54.

18 Pellerier SJ, lezzoni JC, Crabtree TD, et al. Prediction of liver allograft fibrosis after transplantation for hepatitis $C$ virus: persistent elevation of serum transaminase levels versus necroinflammatory activity. Liver Transplant 2000:6:44-5.

19 Hourigan LF, MacDonald GA, Purdie D, et al. Fibrosis in chronic hepatitis C correlates significantly with body mass index and steatosis. Hepatology 1999;29:1215-19.

20 Fujie $\mathrm{H}$, Yotsuyanagi $\mathrm{H}$, Moriya K, et al. Steatosis and intrahepatic hepatitis $\mathrm{C}$ virus in chronic hepatitis. J Med Virol 1999;59:141-5.

21 Hickman IJ, Clouston AD, Macdonald GA, et al. Effect of weight reduction on liver histology and biochemistry in patients with chronic hepatitis C. Gut 2002;51:89-94.

22 Mimms LT, Mosley JW, Hollinger FB, et al. Effect of concurrent acute infection with hepatitis $C$ virus on acute hepatitis $B$ virus infection. $B M J$ 1993;307:1095-7.

23 Hanley JP, Dolan G, Day S, et al. Interaction of hepatitis B and hepatitis C infection in haemophiliacs. Br J Haematol 1993:85:611-12.

24 Weltman MD, Brotodihardjo A, Crewe EB, et al. Coinfection with hepatitis B and $C$ or $B, C$ and delta viruses results in severe chronic liver disease and responds poorly to interferon-alpha. J Viral Hepat 1995;2:39-45.

25 Roudot-Thoraval F, et al. Epidemiological factors affecting the severity of hepatitis $C$ virus-related liver disease: A French survey of 6,664 patients. Hepatology 1997:26:485-90.

26 Villa E, Grottolia A, Buttafoco P, et al. Evidence for hepatitis B virus infection in patients with chronic hepatitis $C$ with and without serological markers of hepatitis B. Dig Dis Sci 1995;40:8-11.

27 Zignego AL, Fontana R, Pulti S, et al. Relevance of inapparent coinfection by hepatitis $B$ virus in alpha interferon-treated patients with hepatitis $C$ virus chronic infection. J Med Virol 1997;51:313-18

28 Cacciola I, Pollicino T, Squadrito G, et al. Occult hepatitis B virus infection in patients with chronic hepatitis C liver disease. N Engl J Med 1999:341:22-6.

29 McOmish F, Yap PL, Dow BC, et al. Geographical distribution of hepatitis C virus genotypes in blood donors: an international survey. J Clin Microbiol 1994;32:884-92

30 Dusheiko G, Schmilovitz WH, Brown D, et al. Hepatitis C virus genotypes: an investigation of type specific differences in geographic origin and disease. Hepatology 1994; 19:13-18.

31 Booth JC, Foster GR, Kumar U, et al. Chronic hepatitis C virus infections: predictive value of genotype and level of viraemia on disease progression and response to interferon alpha. Gut 1995;36:427-32.

32 Silini $E$, Bono F, Cividini A, et al. Differential disribution of hepatitis $C$ virus genotypes in patients with and without liver function abnormalities. Hepatology 1995;21:285-90

33 Preston $\mathrm{FE}$, Jarvis $\mathrm{LM}$, Makris $M$, et al. Heterogeneity of hepatitis $C$ virus genotypes in haemophillia: relationship with chronic liver disease. J Hepatol 1994;21:468-73.

34 McHutchison JG, Gordon SC, Schiff ER, et al. Interferon alfa-2b alone or in combination with ribavirin as initial treatment for chronic hepatitis $C$. N Engl J Med 1998;339:1485-92.

35 Piperno A, D'Alba R, Fargion S, et al. Liver iron concentration in chronic viral hepatitis: a study of 98 patients. Eur J Gastroenterol Hepatol 1995;7:1203-8.

36 Rigamonti C, Andorno S, Maduli E, et al. Iron, hepatic stellate cells and fibrosis in chronic hepatitis C. Eur J Clin Invest 2002;32(suppl 1):28-35.

37 Beinker NK, Vogt MD, Arendse M, et al. Threshold effect of liver iron content on hepatic inflammation and fibrosis in hepatitis B and C. J Hepatol $1996 ; 25: 633-8$ 